\title{
Clinical features of intracranial vestibular schwannomas
}

\author{
XIANG HUANG ${ }^{*}$, JIAN XU* ${ }^{*}$ MING XU, LIANG-FU ZHOU, \\ RONG ZHANG, LIQIN LANG, QIWU XU, PING ZHONG, \\ MINGYU CHEN, YING WANG and ZHENYU ZHANG \\ Department of Neurosurgery, Huashan Hospital, Shanghai Medical College, \\ Fudan University, Shanghai 200040, P.R. China
}

Received July 5, 2012; Accepted October 2, 2012

DOI: $10.3892 / 01.2012 .1011$

\begin{abstract}
The aim of the present study was to discuss the clinical features of intracranial vestibular schwannomas and to evaluate the symptoms and signs as well as their correlation with tumor extension. The records of 1,009 patients who were treated in Shanghai Huashan Hospital were reviewed retrospectively. According to the Samii classification of 1997, the patients were grouped into a T3 and a T4 group based on the radiological findings. We focused our analysis on the incidence of subjective disturbances versus objective morbidity, and symptomatology versus tumor size and extension. Of the 1,009 cases, 424 patients $(42.0 \%)$ were defined as T3 while 585 patients $(58 \%)$ were defined as $\mathrm{T} 4$. The most frequent clinical symptoms were hearing loss (85.8\%), facial numbness (48.9\%), ataxia (44.6\%), tinnitus (40.1\%), deafness (26.3\%) and facial paralysis $(21.1 \%)$. The ratios of gender, vertigo and facial paralysis were significantly different between the $\mathrm{T} 3$ and $\mathrm{T} 4$ groups $(\mathrm{P}<0.05)$; however, none of the clinical symptoms had a positive likelihood ratio (PLR) greater than 10 for T4 prediction. The most frequent cranial nerve disturbance was associated with the cochlear nerve $(92.6 \%)$ and trigeminal nerve $(53.5 \%)$. Disturbance of the facial nerve was more severe in $\mathrm{T} 3$ than $\mathrm{T} 4$ patients $(\mathrm{P}<0.05)$. Hearing deficit, facial paresthesia, ataxia and tinnitus are key symptoms of huge vestibular schwannomas. Cochlear, trigeminal and facial nerves were the most commonly affected cranial nerves in cases of large tumors. Gender and tumor size were associated with tumor extension. Although the predictive value was limited, the symptoms of vertigo, facial paralysis and hearing loss may be indicators of tumor growth.
\end{abstract}

Correspondence to: Dr Ping Zhong, Department of Neurosurgery, Huashan Hospital, Shanghai Medical College, Fudan University, 12 Wulumuqi Zhong Road, Shanghai 200040, P.R. China

E-mail: xiang.h.huang@gmail.com

*Contributed equally

Key words: vestibular schwannomas, clinical feature, alarm feature, tumor growth

\section{Introduction}

Comprising approximately $6 \%$ of all intracranial tumors, vestibular schwannomas, also known as acoustic neuromas, are a common disease found in the region of the cerebellopontine angle (1). Although many data have been gathered, numerous phenomena encountered in this disease are not yet understood. Neurosurgeons have established that hearing loss, deafness and tinnitus are common clinical presentations for this disease; however, larger case studies are required to identify the most common and significant clinical features of the disease. Furthermore, controversy remains as to the clinical features that predict the development of tumors. It is not clear how the various symptoms, duration and sequence of occurrence correlate with the size and extension of the tumor or with the actual objective cranial nerve damage.

Currently, more and more research is focusing on conservative management aimed at preventing the further growth of lesions (2). A 'watch-wait-rescan' strategy appears to be justified in numerous patients. However, there is a potential burden of worry for individuals with an acoustic neuroma managed with a watch-wait-rescan strategy when they are aware that their tumor may progress at any time. Thus, distinguishing individual patients whose tumors will progress and pose a threat from those whose tumors are likely to remain dormant or even regress is central to the current management of these patients. Increasingly, evidence of tumor growth has become the defining criterion for intervention. Consequently, an analysis of these epidemiological aspects of alarm features is necessary before formulating an optimal diagnostic and therapeutic protocol.

In recent years, a number of studies concerning vestibular schwannomas have focused on cranial nerve preservation rather than description of clinical presentation. The 1,009 cases treated in Shanghai Huashan Hospital between 1999 and 2009 offer an opportunity to detail the clinical features and management of these tumors. In this study, we focus on the clinical features of intracranial vestibular schwannomas, and evaluate the symptom and signs as well as their correlation with tumor extension.

\section{Patients and methods}

Patient population. A retrospective review of medical records from 1999 to 2009 identified 1,009 patients with intracranial 
vestibular schwannomas. Tumor removal was performed using a sub-occipital approach for all cases and the received histology results were verified. The study was approved by the ethics committee of the Institution of Neurology, Fudan University, Shanghai, China. Written informed consent was obtained from the patient's family.

Clinical manifestation. In this study, we focused on the clinical evaluation that was based in part on records of patients' complaints and neurological examinations in the following areas: i) general state of health; ii) involvement of the cranial nerves; iii) involvement of the cerebellum and the cerebrum; and iv) duration and sequence of symptoms.

The objective investigation for all patients preoperatively included bone window computed tomography (CT), contrast-enhanced magnetic resonance imaging (MRI) and brainstem electric response audiometry, which were performed before surgery and 1 to 2 weeks postoperatively in each case.

Tumor size and classification. Tumor sizes were measured in the axial plane, considering intra- and extra-meatal tumor extension. The data were recorded for each operative protocol. Tumors larger than $30 \times 20 \mathrm{~mm}$ were defined as large; small tumors measured less than $30 \times 20 \mathrm{~mm}$. Tumor extension classes were described as follows: T1, purely intrameatal; T2, intra-extrameatal; T3, filling the cerebellopontine cistern or reaching the brain stem; $\mathrm{T} 4$ compressing the brain stem or severely dislocating the brain stem and compressing the fourth ventricle.

Statistical analysis. Categorical data were compared by the $\chi^{2}$ test with continuity correction if appropriate. Continuous variables are expressed as the mean $\pm \mathrm{SD}$, and were compared using the Student's t-test. The diagnostic values of symptoms including sensitivity, specificity, positive predictive value (PPV), negative predictive value (NPV), positive likelihood ratio (PLR), negative likelihood ratio (NLR) and their 95\% CIs were calculated and compared between classes T3 and T4. Two-tailed P-values $<0.05$ were considered to indicate a statistically significant difference. Statistical analysis was performed with the Stata software package (version 10.0).

\section{Results}

General patient data. In this group of medical cases, the male to female ratio was 475:534 and the age range was $12-80$ years (median, 47.6 \pm 12.2 ). Most of the patients were 40-60 years of age, accounting for $57.2 \%$ of the total patients. There were 11 patients younger than 20 years of age, making up only $1.1 \%$ of cases. There were $277(27.5 \%)$ and 144 (14.2\%) patients belonging to the $20-40$ and $60-80$ age groups, respectively. The age and gender distribution revealed that middle-aged females between 40 and 60 years of age were a high-risk population in this disease, accounting for $61.6 \%$ of total female patients; this figure was $32.7 \%$ higher than that observed for male patients in the same age group (Fig. 1).

Tumor extension analysis revealed that $42 \%$ of tumors belonged to class $\mathrm{T} 3$ and $58 \%$ to class T4. A total of 53\% of tumors were located on the right and $47 \%$ on the left. Tumor length and width were significantly different between these

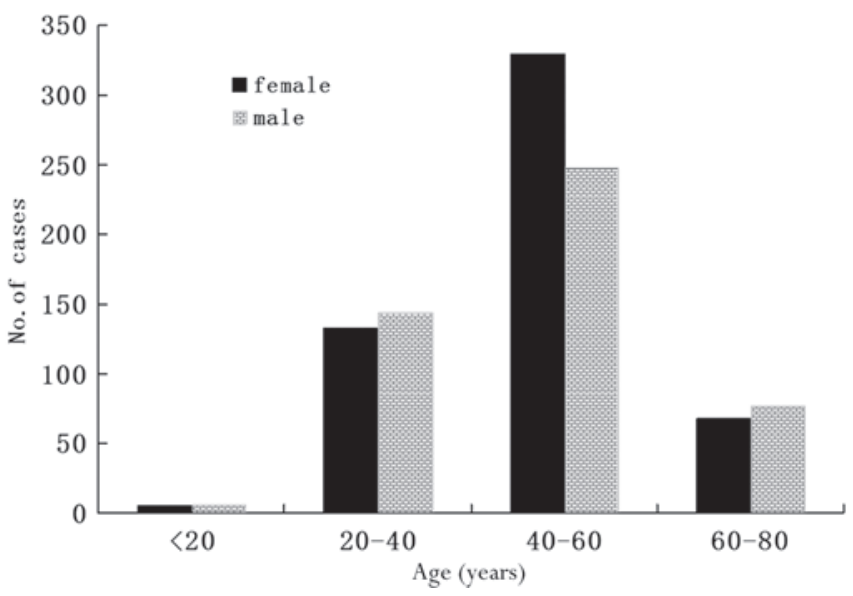

Figure 1. Age and gender distribution of 1,009 intracranial vestibular schwannoma patients.

classes, with a mean length and width of $30 \times 25 \mathrm{~mm}$ in class $\mathrm{T} 3$ and $44 \times 36 \mathrm{~mm}$ in class $\mathrm{T} 4$.

Symptoms and signs. Among the 1,009 patients, the most frequent clinical symptoms were hearing loss (85.8\%), facial paresthesia (48.9\%), instability of gait (44.6\%), tinnitus (40.1\%), deafness $(26.3 \%)$ and facial paralysis $(21.1 \%)$. During preoperative physical examination, absent corneal reflex was the most common sign, observed in $15.2 \%$ of the 1,009 patients. However, Romberg sign and abduction disorder were both uncommon, observed in $<2 \%$ of patients (Table I).

The most frequent cranial nerve disturbance was related to the cochlear nerve (92.6\%) and trigeminal nerve (53.5\%). There were only 17 (1.7\%) cases involving the abducent nerve, which became the minimal impact nerve by tumors (Table II).

Cochlear nerve symptoms accounted for the most frequent complaints (Table II). A total of 648 patients noticed some degree of hearing deficit and 265 patients experienced deafness. Tinnitus occurred at an incidence of 43.7 and $38.1 \%$ in hypacusis and deaf patients, respectively. The incidence of facial paralysis and instability of gait were significantly different between the two groups $(\mathrm{P}<0.05)$.

Difference between class T3 and T4. The average ages of T3 and T4 patients were 47.9 and 47.3 years of age, respectively ( $\mathrm{P}>0.05)$. There were 209 female patients in T3, accounting for $49.3 \%$ of this class. However, the female incidence in class T4 was much higher, making up 55.6\% of this stage $(\mathrm{P}<0.05)$. Twenty-one (5.0\%) patients in class T3 suffered tumor hemorrhage compared with $16(2.7 \%)$ patients in T4; this difference was not significant $(\mathrm{P}>0.05)$. A total of $143(33.7 \%)$ cystic tumors were observed in class T3 and $171(29.2 \%)$ were observed in $\mathrm{T} 4(\mathrm{P}>0.05)$.

There were 55 (13.0\%) patients who suffered from vertigo in T3 compared with 105 (17.9\%) in T4. The incidence of facial paralysis showed a correlation with tumor extension; patients with larger tumors had higher rates of facial paralysis. The incidence was $18.8 \%$ in $\mathrm{T} 3$ and $23.3 \%$ in $\mathrm{T} 4$. We therefore concluded that the ratio of gender, vertigo, facial paralysis and absent corneal reflex were significantly different between class $\mathrm{T} 3$ and $\mathrm{T} 4(\mathrm{P}<0.05$; Table I). 
Table I. Major preoperative symptoms and signs of 1,009 cases of intracranial vestibular schwannomas.

\begin{tabular}{|c|c|c|c|c|}
\hline Symptoms and signs & Total $(\%)$ & $\mathrm{T} 3(\%)$ & $\mathrm{T} 4(\%)$ & P-value \\
\hline Headache & $259(25.7)$ & $107(25.2)$ & $152(26.0)$ & 0.789 \\
\hline Hypacusis & $866(85.8)$ & $357(84.2)$ & $509(87.0)$ & 0.206 \\
\hline Hearing loss & $265(26.3)$ & $108(25.5)$ & $157(26.8)$ & 0.627 \\
\hline Tinnitus & $405(40.1)$ & 177 (41.7) & $228(39.0)$ & 0.375 \\
\hline Vertigo & $160(15.9)$ & $55(13.0)$ & 105 (17.9) & 0.033 \\
\hline Instability of gait & $450(44.6)$ & $203(47.9)$ & $247(42.2)$ & 0.074 \\
\hline Facial paralysis & $213(21.1)$ & $77(18.8)$ & $137(23.3)$ & 0.035 \\
\hline Facial paresthesia & $493(48.9)$ & $200(47.2)$ & $293(50.1)$ & 0.360 \\
\hline Ear pain & $4(0.4)$ & $1(0.2)$ & $3(0.5)$ & 0.854 \\
\hline Bucking & $99(9.8)$ & $39(9.2)$ & $60(10.3)$ & 0.577 \\
\hline Hoarseness & $18(1.8)$ & $9(2.1)$ & $9(1.5)$ & 0.489 \\
\hline Visual disorder & $82(8.1)$ & $35(8.3)$ & $47(8.0)$ & 0.899 \\
\hline Movement disorder & $41(4.1)$ & $19(4.5)$ & $22(3.8)$ & 0.567 \\
\hline Nausea and vomiting & $57(5.6)$ & $23(5.4)$ & $34(5.8)$ & 0.792 \\
\hline Romberg sign & $20(2.0)$ & $9(2.1)$ & $11(1.9)$ & 0.785 \\
\hline Nystagmus & $52(5.2)$ & $22(5.2)$ & $30(5.1)$ & 0.966 \\
\hline Absent corneal reflex & $153(15.2)$ & $78(18.4)$ & $75(12.8)$ & 0.015 \\
\hline Mastication disorder & $26(2.6)$ & $14(3.3)$ & $12(2.1)$ & 0.216 \\
\hline Abduction disorder & $17(1.7)$ & $6(1.4)$ & $11(1.9)$ & 0.571 \\
\hline
\end{tabular}

Table II. Preoperative cranial nerve disturbance of 1,009 cases of intracranial vestibular schwannomas.

\begin{tabular}{|c|c|c|c|c|c|}
\hline Nerve & Symptom/sign & Total $(\%)$ & $\mathrm{T} 3(\%)$ & $\mathrm{T} 4(\%)$ & P-value \\
\hline $\mathrm{V}$ & Trigeminal nerve disturbance & $540(53.5)$ & $222(52.4)$ & $318(54.4)$ & 0.529 \\
\hline VI & Abduction disorder & $17(1.7)$ & $6(1.4)$ & $11(1.9)$ & 0.571 \\
\hline VII & Facial nerve disturbance & $230(22.8)$ & $67(14.9)$ & $163(27.8)$ & 0.035 \\
\hline VIII & Hearing deficits & $934(92.6)$ & $393(92.7)$ & $541(92.5)$ & 0.900 \\
\hline VIII & Vestibular disturbance & $209(20.7)$ & $76(17.9)$ & $133(22.7)$ & 0.063 \\
\hline IX-XII & Caudal cranial nerve deficits & $153(15.2)$ & $67(15.8)$ & $86(14.7)$ & 0.630 \\
\hline
\end{tabular}

As mentioned previously, the most frequent cranial nerve disturbance was related to the cochlear nerve $(92.6 \%)$ and trigeminal nerve $(53.5 \%)$. Facial nerve disturbance was more severe in class T3 than T4. There were $67(14.9 \%)$ patients in $\mathrm{T} 3$ and 163 (27.8\%) in T4 for facial nerve disturbance $(\mathrm{P}<0.05$; Table II).

Next, we also used diagnostic test parameters, including sensitivity, specificity, PPV, NPV, PLR, NLR and their 95\% CIs, to identify the differences between T3 and T4 (Table III). However, none of the clinical symptoms had a PLR $>10$ for T4 prediction.

\section{Discussion}

General findings. Intracranial vestibular schwannomas comprise approximately $85 \%$ of tumors in the region of the cerebellopontine angle. Approximately 10 tumors are diagnosed per million individuals each year (1). The tumors may cause dysfunction of the cochlear nerve, including unilateral hypacusis, hearing loss, tinnitus and vertigo.
Diplopia may occur due to abducens palsy. If the trigeminal nerve is involved, facial paresthesia, facial pain, absent corneal reflex and mastication disorder may occur. Facial paralysis or spasms are uncommon. Large lesions may lead to dysphonia, dysarthria and dysphagia due to involvement of the IX and X cranial nerve. Impaired coordination and upper motor neuron signs in the limbs are symptoms of cerebellar and/or brain stem compression. Certain patients experience pain localized in the ear/mastoid region and occasionally non-localized headache $(3,4-9)$. Among our 1,009 patients, the most frequent clinical symptoms were hearing loss $(85.8 \%)$, facial paresthesia (48.9\%), instability of gait (44.6\%), tinnitus (40.1\%), deafness (26.3\%) and facial paralysis $(21.1 \%)$. During preoperative physical examination, absent corneal reflex was the most common sign, observed in $15.2 \%$ of the patients. However, Romberg sign and abduction disorder were both uncommon, appearing in less than $2 \%$ of patients (Table I). The most frequent cranial nerve disturbance was related to the cochlear nerve $(92.6 \%)$ and trigeminal nerve $(53.5 \%)$. 


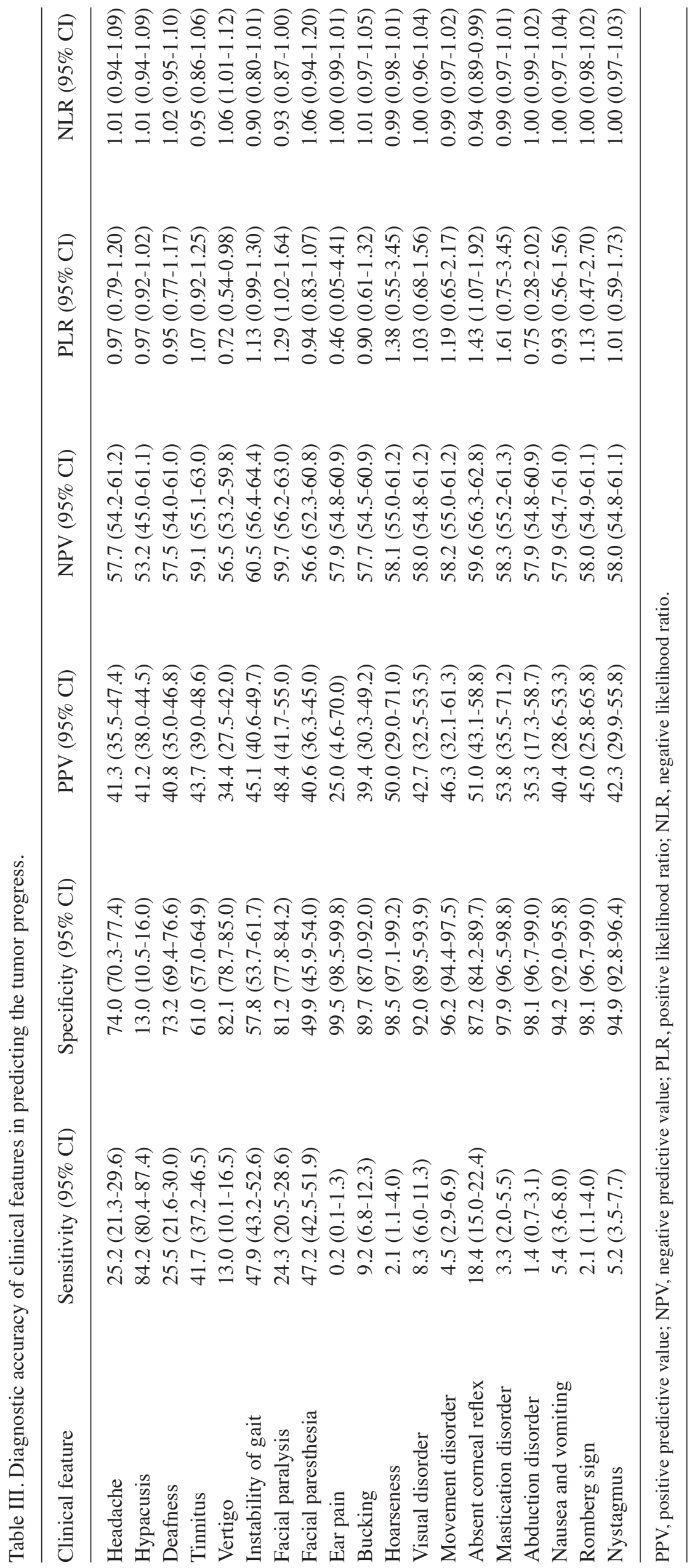


Table IV. Parameters and correlation with preoperative hearing function.

\begin{tabular}{lccr}
\hline Parameter & Hypacusis & Hearing loss & P-value \\
\hline Mean age (years) & 47.8 & 48.8 & 0.235 \\
Tumor diameter (mm) & 3.12 & 3.15 & 0.701 \\
Headache (\%) & $166(25.6)$ & $69(26.0)$ & 0.895 \\
Trigeminal nerve & $354(54.6)$ & $142(53.6)$ & 0.774 \\
disturbance (\%) & & & \\
Tinnitus (\%) & $283(43.7)$ & $101(38.1)$ & 0.122 \\
Vertigo (\%) & $107(16.5)$ & $37(14.0)$ & 0.337 \\
Instability of gait (\%) & $281(43.4)$ & $139(52.5)$ & 0.012 \\
Facial paralysis (\%) & $117(18.1)$ & $79(29.8)$ & $<0.001$ \\
Movement disorder (\%) & $22(3.4)$ & $11(4.2)$ & 0.579 \\
Romberg sign (\%) & $12(1.9)$ & $8(3.0)$ & 0.274 \\
\hline
\end{tabular}

Both conservative management and post-operative follow up are needed to observe the tumor extension (10-12) since certain vestibular schwannomas present with rapid growth that may lead to serious morbidity and even mortality if left untreated. In the present study, we attempted to find the clinical alarm features for predicting tumor progress. This may guide clinical conservative management of vestibular schwannomas and become the first step for evaluating the tumor extension, which must be followed by CT and MRI.

\section{Factors associated with tumor extension}

Age. Matthies and Samii analyzed 1,000 vestibular schwannomas and found an inverse correlation between patients' ages and the degree of tumor extension (younger age correlates with larger tumors) (13). However, a meta-analysis of 10 studies and 620 patients did not reveal age to be a predictive factor for tumor growth (14). In another meta-analysis of 555 patients in 13 studies, no statistically significant difference was found with regard to the mean age and mean initial size between growth and non-growth groups (15). In a systematic review of 1,340 patients, no correlation was found between the proportion of tumors that grew and patient age at diagnosis or follow-up duration (16). In our group, the average ages of T3 and T4 patients were 47.9 and 47.3 years $(\mathrm{P}>0.05)$ The data did not reveal a correlation between age and tumor extension. Similarly, Rosenberg concluded from a large retrospective study that there was no significant correlation between tumor size and patient age (17).

Gender. Ogawa et al found tumor extension to be correlated with gender (18). On the basis of this analysis, female patients had larger tumors at presentation and their tumors grew faster. Beenstock found that probability of stability was independent of gender (11). However, Stangerup et al analyzed 552 patients and found no correlation between gender and tumor growth (19). Our analysis of age and gender distribution revealed that middle-aged females ranging from 40-60 years of age were most at risk, accounting for $61.6 \%$ of total female patients and $32.7 \%$ higher than for male patients in the same age group. There were 209 female patients in class T3, accounting for $49.3 \%$ of this class. However, the female incidence in T4 was much higher, making up 55.6\% of this class. The present study supports the finding of Ogawa et al that female patients had larger tumors and are possibly a high-risk population for vestibular schwannomas.

Size. In a review of 119 patients, Fucci et al found that tumors larger than $20 \mathrm{~mm}$ at presentation are more likely to exhibit rapid growth than those smaller than $20 \mathrm{~mm}$ (20). Walsh et al, in an 11-year retrospective study, showed that small tumor confined to the canaliculus did not exhibit significant growth compared with tumors in the cerebellopontine angle cistern (21). In our study of 1,009 cases, the tumor length and width were significantly different among classes T3 and T4, with a mean length and width of $30 \times 25 \mathrm{~mm}$ in T3 and $44 \times 36 \mathrm{~mm}$ in $\mathrm{T} 4$.

Symptoms and signs predicting tumor progress

Vertigo. In this study, we found the incidence of vertigo was significant higher in class $\mathrm{T} 3$ than in class $\mathrm{T} 4(\mathrm{P}<0.05)$ The specificity for vertigo was $82.1 \%$ in the diagnostic assessment (Table III). Similarly, Artz et al analyzed 234 vestibular schwannoma patients and found vertigo was a predictor for the growth of sporadic tumors (22). Theoretically, vertigo may occur due to vestibular nerve dysfunction as well as brain stem compression. Tumors with a diameter larger than $20 \mathrm{~mm}$ in the cerebellopontine angle are likely to reach the brain stem, which obviously adds to the symptoms of vertigo. In a recent study, the theory hypothesizes that endolymph draining too rapidly from the cochlear duct (pars inferior) causes attacks of vertigo. The endolymph overfills the endolymphatic sinus and overflows into the utricle (pars superior), stretching the cristae of the semicircular canals and causing the attacks of vertigo (23). We speculate that the tumor extension will affect the vestibular nerve function in vessels and lymphatic backflow which cause vertigo. A compensatory mechanism influenced by vision and the contralateral vestibular apparatus possibly contributes to delaying the onset of vertigo.

Facial paralysis. Another phenomenon of these 1,009 cases was that the incidence of facial paralysis showed a correlation with tumor extension; patients with larger tumors had higher rates of facial paralysis. The incidence was $18.8 \%$ in class T3 and $23.3 \%$ in class $\mathrm{T} 4(\mathrm{P}<0.05)$.

Notably, facial paralysis at admittance was a result of two factors; firstly, a large number of patients had undergone previous partial tumor resection elsewhere, and secondly, a high rate of large tumors had brain stem compression and therefore caused elongation of the facial nerve. Samii and Matthies analyzed 1,000 patients in 1997 (24). Of the patients with preoperative facial nerve paresis, $1 \%$ were assigned to class $\mathrm{T} 1,10 \%$ to $\mathrm{T} 2,23 \%$ to $\mathrm{T} 3$ and $55 \%$ to $\mathrm{T} 4$. This study demonstrated that this symptom is largely dependent on a patient's ability to differentiate and perceive such slight changes. A systematic search for taste disturbances should be included in history taking; in case of doubt or suspicion, electrogustometry is capable of detecting disorders early.

Notably, although the facial nerve may be thinly stretched by the tumor, facial nerve weakness is not a prominent sign in many cases preoperatively $(25,26)$. When facial nerve function is compromised it usually has an insidious onset over several months and is not a sudden event. 
Hearing loss. It remains unclear whether hearing loss predicts tumor extension. Artz et al analyzed 234 vestibular schwannomas and concluded that the predictor factors for growth are vertigo, no sudden onset of hearing loss and short duration of hearing loss (22).

Tschudi et al found that patients with progressive hearing loss as a first symptom had a significantly lower tumor growth than those presenting with tinnitus, sudden hearing loss and vertigo (27). Other studies, however, revealed no significant differences in this symptom between patients with growing tumors and non-growing tumors $(28,29)$.

In our research, hearing loss was one of the most frequent symptoms in vestibular schwannomas. Cochlear nerve symptoms accounted for the most frequent complaints (Table II). There were 648 patients who noticed some degree of hearing deficit and 265 patients experienced deafness. Although the proportion of patients suffering hearing loss was similar in classes T3 and T4, the rates of vestibular disturbances most often occur as some unsteadiness while walking and facial disturbances, such as facial paralysis. These factors are predictors of tumor extension, as mentioned previously, and their incidence was significantly higher in deaf than in hearing patients $(\mathrm{P}<0.05$; Table IV). This evidence supports the hypothesis that hearing loss may be a predictive factor of tumor extension.

Conclusions and future considerations. Hearing deficit, facial paresthesia, ataxia and tinnitus are the most frequent and significant symptoms of huge vestibular schwannomas. The cochlear, trigeminal and facial nerves are those most commonly affected by large tumors. Gender and tumor size are associated with tumor extension. Although the predictive value was limited, the symptoms of vertigo, facial paralysis and hearing loss may be alarm features predicting tumor growth. The symptoms and signs are the first step in determining tumor extension. Further methods, including CT and MRI, are required to evaluate tumor progress.

\section{Acknowledgements}

The authors thank the surgeons and pathologists at the Department of Neurosurgery in Huashan Hospital. Professor Ying Mao provided valuable advice for this study. This study was presented in part at the 14th European Congress of Neurosurgery (Rome, Italy; October 9-14, 2011) (30).

\section{References}

1. Evans DG, Moran A, King A, Saeed S, Gurusinghe N and Ramsden R: Incidence of vestibular schwannoma and neurofibromatosis 2 in the north west of england over a 10-year period: higher incidence than previously thought. Otol Neurotol 26: 93-97, 2005.

2. Nikolopoulos TP, Fortnum H, O'Donoghue G and Baguley D: Acoustic neuroma growth: a systematic review of the evidence. Otol Neurotol 31: 478-485, 2010.

3. Zhou LF, Chen XC and Shi YQ: Modern Neurosurgery. Fudan Press, Shanghai, 2001.

4. Samii M, Gerganov V and Samii A: Improved preservation of hearing and facial nerve function in vestibular schwannoma surgery via the retrosigmoid approach in a series of 200 patients. J Neurosurg 105: 527-535, 2006.

5. Goksu N, Bayazit Y and Kemaloglu Y: Endoscopy of the posterior fossa and endoscopic dissection of acoustic neuroma. Neurosurg Focus 6: e15, 1999.
6. Tringali S, Bertholon P, Chelikh L, Jacquet C, Prades JM and Martin C: Hearing preservation after modified translabyrinthine approach performed to remove a vestibular schwannoma. Ann Otol Rhinol Laryngol 113: 152-155, 2004.

7. Sanna M, Khrais T, Russo A, Piccirillo E and Augurio A: Hearing preservation surgery in vestibular schwannoma: the hidden truth. Ann Otol Rhinol Laryngol 113: 156-163, 2004.

8. Chee GH, Nedzelski JM and Rowed D: Acoustic neuroma surgery: the results of long-term hearing preservation. Otol Neurotol 24: 672-676, 2003.

9. Brackmann DE, Owens RM, Friedman RA, et al: Prognostic factors for hearing preservation in vestibular schwannoma surgery. Am J Otol 21: 417-424, 2000.

10. Lassaletta L, Fontes L, Melcon E, Sarria MJ and Gavilan J: Hearing preservation with the retrosigmoid approach for vestibular schwannoma: Myth or reality? Otolaryngol Head Neck Surg 129: 397-401, 2003.

11. Beenstock M: Predicting the stability and growth of acoustic neuromas. Otol Neurotol 23: 542-549, 2002.

12. Yamakami I, Oka N and Yamaura A: Intraoperative monitoring of cochlear nerve compound action potential in cerebellopontine angle tumour removal. J Clin Neurosci 10: 567-570, 2003.

13. Matthies C and Samii M: Management of 1000 vestibular schwannomas (acoustic neuromas): clinical presentation. Neurosurgery 40: 1-9 (discussion 9-10), 1997.

14. Smouha EE, Yoo M, Mohr K and Davis RP: Conservative management of acoustic neuroma: a meta-analysis and proposed treatment algorithm. Laryngoscope 115: 450-454, 2005.

15. Selesnick SH and Johnson G: Radiologic surveillance of acoustic neuromas. Am J Otol 19: 846-849, 1998.

16. Yoshimoto Y: Systematic review of the natural history of vestibular schwannoma. J Neurosurg 103: 59-63, 2005.

17. Rosenberg SI: Natural history of acoustic neuromas. Laryngoscope 110: 497-508, 2000.

18. Ogawa K, Kanzaki J, Ogawa S, Yamamoto M, Ikeda S and Shiobara R: The growth rate of acoustic neuromas. Acta Otolaryngol Suppl 487: 157-163, 1991.

19. Stangerup SE, Caye-Thomasen P, Tos M and Thomsen J: The natural history of vestibular schwannoma. Otol Neurotol 27: 547-552, 2006.

20. Fucci MJ, Buchman CA, Brackmann DE and Berliner KI: Acoustic tumor growth: Implications for treatment choices. Am J Otol 20: 495-499, 1999.

21. Walsh RM, Bath AP, Bance ML, Keller A, Tator $\mathrm{CH}$ and Rutka JA: The role of conservative management of vestibular schwannomas. Clin Otolaryngol Allied Sci 25: 28-39, 2000.

22. Artz JC, Timmer FC, Mulder JJ, Cremers CW and Graamans K: Predictors of future growth of sporadic vestibular schwannomas obtained by history and radiologic assessment of the tumor. Eur Arch Otorhinolaryngol 266: 641-646, 2009.

23. Gibson WP: Hypothetical mechanism for vertigo in Meniere's disease. Otolaryngol Clin North Am 43: 1019-1027, 2010.

24. Samii M and Matthies C: Management of 1000 vestibular schwannomas (acoustic neuromas): the facial nerve - preservation and restitution of function. Neurosurgery 40: 684-694 (discussion 694-685), 1997.

25. Arts HA, Telian SA, El-Kashlan H and Thompson BG: Hearing preservation and facial nerve outcomes in vestibular schwannoma surgery: results using the middle cranial fossa approach. Otol Neurotol 27: 234-241, 2006.

26. Martin TP: Conservative management of acoustic neuroma: a meta-analysis and proposed treatment algorithm. Laryngoscope 115: 1704, 2005.

27. Tschudi DC, Linder TE and Fisch U: Conservative management of unilateral acoustic neuromas. Am J Otol 21: 722-728, 2000.

28. Flint D, Fagan P and Panarese A: Conservative management of sporadic unilateral acoustic neuromas. J Laryngol Otol 119: 424-428, 2005.

29. Herwadker A, Vokurka EA, Evans DG, Ramsden RT and Jackson A: Size and growth rate of sporadic vestibular schwannoma: predictive value of information available at presentation. Otol Neurotol 26: 86-92, 2005.

30. Huang $\mathrm{X}, \mathrm{Xu} \mathrm{J}$ and Zhong P: The clinical feature of intracranial vestibular schwannomas-A retrospective review of 1009 vestibular schwannomas in single hospital. Acta Neurochirurgica 153: 1833-1905, 2011 